\title{
The Role of Social Cognition in Medical Decision Making with Asian American Patients
}

\author{
Ivy K. Ho ${ }^{1}$ (D) - Jason S. Lawrence ${ }^{1}$
}

Received: 9 March 2020 / Revised: 5 September 2020 / Accepted: 7 September 2020 / Published online: 14 September 2020

(C) W. Montague Cobb-NMA Health Institute 2020

\begin{abstract}
Asian Americans, the fastest growing racial group in the USA, face a host of major health disparities. There are several reasons for these disparities, and one possible contributor is provider perceptions of Asian Americans, which in turn can affect their medical decision making when treating Asian American patients. There is evidence for the influence of provider perceptions on medical decision making among patients of other racial minority groups; however, literature on Asian American patients is lacking. The present paper addresses this gap in the literature by using social cognitive theory to outline the mechanisms through which provider perception of Asian American patients can affect diagnostic and treatment decisions. These mechanisms include stereotypes and implicit biases, illusory correlations, and cognitive load. Recommendations for future research and policy development are provided.
\end{abstract}

Keywords Asian Americans $\cdot$ Health disparities $\cdot$ Social cognition $\cdot$ Medical decision making

\section{Introduction}

Asian Americans, the fastest-growing racial group in the USA, comprise approximately $6 \%$ of the country's population [1]. Contrary to popular perception that Asian Americans are a homogenous monolith, there are actually at least 19 distinct Asian ethnic groups [2], which can be broadly categorized as East, South, and Southeast Asian. Some Asians in the USA trace their roots to Filipinos who arrived in California in the 1500 s, while others are new arrivals who immigrated only recently [1]. Asians came to the USA as slaves, refugees, asylum seekers, and voluntary immigrants. With one out of seven Asian immigrants being undocumented, Asians are the fastest growing group of undocumented immigrants, making up approximately 1.7 million of the 11 million undocumented immigrants in 2017 [3].

In addition to being diverse in terms of ethnic background and immigration history, Asian Americans also differ from

Ivy K. Ho

Ivy_Ho@uml.edu

Jason S. Lawrence

Jason_Lawrence@uml.edu

1 Department of Psychology, University of Massachusetts Lowell, 113 Wilder Street, Suite 300, Lowell, MA 01854-3059, USA one another with regard to socioeconomic status. Southeast Asian Americans have the lowest income and lowest educational attainment compared with Asians of other subgroups [4]. Compared with other major racial groups in the USA, Asian Americans have the largest income disparity, with Asians in the top $10 \%$ income bracket earning 10.7 times more than those in the bottom $10 \%$ [5].

Despite the growing population of Asians in the USA, their healthcare needs are overlooked. Asian Americans face an array of health disparities. They account for more than half of all Hepatitis B [6] and more than one-third of tuberculosis patients in the USA [7]. Asian Americans are at least twice as likely as White Americans to suffer from stomach and liver cancers [8], and Asian American women are more likely to die from breast and cervical cancers than White women [9]. The mortality burden of cardiovascular disease among Asian American ethnic groups is higher than that of non-Hispanic White Americans [10]. Compared with White Americans, Asian Americans experience worse quality of care in the 47 out of 172 quality measures conducted by the Agency for Healthcare Research and Quality, with the greatest disparities found in measures of patient-centered care [11]. Finally, although the rate of type 2 diabetes among Asian Americans is comparable with that of Black and Hispanic Americans (21\%), about $50 \%$ of diabetes cases among Asian Americans are undiagnosed [12]. It is important to note ethnic differences in these health disparities. For example, among the 
Asian ethnic groups, cervical cancer mortality is highest among Vietnamese, Cambodian and Korean Americans, and Chinese and Filipinos have a high prevalence of liver cancer [13].

Although there are many reasons for these health disparities, including poverty, low health literacy, and harmful health behaviors, one possible contributor to the poor health among Asian Americans is provider perceptions and behaviors. This possibility seems likely given the evidence that other minorities (Black, Latinx, Hispanic, and Native American patients) receive poorer healthcare than White patients. Indeed, racial and ethnic minority patients receive poorer care than do White patients across a variety of illness domains, including transplant, cardiac diseases, pain management, and cancer [14, 15], and these discrepancies occur across various stages of the diagnosis and treatment process. This is the case even after taking into account patients' access-related factors, such as income and health insurance [16]. Furthermore, when race intersects with gender, medical students attribute lower health status to a Black woman than to a White man presenting with the same symptoms [17]. Many have argued that these differences in treatment decisions can be attributed to a lack of cultural competence and a lack of awareness of one's stereotypes and other cognitive biases toward non-dominant, minority groups [18-20].

Despite the evidence that providers' stereotypes and other biases lead to poorer medical treatment for Black, Hispanic, and Native American patients, little is known about whether or how stereotypes impact the medical care of Asian American patients. It is likely that given the evidence that other minority groups experience bias in healthcare setting, Asian Americans also experience a similar bias. Asian American patients are more likely than White American patients to report that their healthcare providers did not understand their backgrounds, did not involve them in healthcare decisions as much as they wanted, or did not treat them with respect [21]. There is little research on healthcare providers' stereotypes of Asians and how those stereotypes impact their medical care. However, we do know that Asian patients report that they sense some bias in their healthcare providers. It is possible that healthcare providers treat Asian Americans differently due to their perceptions and stereotypes of this population, raising the issue that providers' perceptions can have an impact on their interactions with Asian American patients, subsequent diagnoses, and treatment decisions.

The purpose of this paper is to apply social cognition to explain how providers' perceptions may influence treatment of Asian American patients. Betancourt and AnanehFirempong [18] had previously used social cognitive theory to explore how providers' medical decisions are influenced by social information such as the group membership (such as race, socioeconomic status, and gender) of their patients and concluded that medical decisions are affected by non-clinical factors, the key of which is provider stereotypes. Although we had noted that Asian Americans are ethnically diverse, the existing literature overlooks these distinctions; therefore, our review below pertains to the general Asian American population.

\section{Social and Cognitive Biases}

\section{Stereotypes and Implicit Biases}

The US Department of Health and Human Services Health Resources \& Services Administration [22] mentioned "stereotyping" or "stereotype" 18 times in their guidebook on cultural competence education for medical schools. In their Tool for Assessing Cultural Competence Training, the Association of American Medical Colleges [23] includes "understanding the impact of stereotyping on medical decisionmaking" as one of the five content domains of cultural competence for medical providers. Yet, even if provider stereotypes were addressed in cultural competence training, stereotyping often occurs outside of the providers' awareness [24] and can influence providers' interpretation of minority patients' behaviors and result in differential treatment of these patients.

Implicit biases are stereotypes that occur outside of one's awareness and can influence one's behaviors and decisions. For example, physicians who reported no explicit biases against Black patients, and who reportedly did not perceive Black patients to be less cooperative, nonetheless demonstrated implicit biases against Black patients and the implicit belief that these patients were less cooperative [25]. Providers' implicit biases result in differential treatment decisions for Black patients compared with White patients, such as in pain management [26] and thrombolysis [25].

Although much research on stereotyping and implicit bias has focused on Black Americans, negative attitudes against Asian Americans are also prevalent [27]. More than 25\% of Americans reported very negative attitudes toward Chinese Americans, with another $43 \%$ reporting somewhat negative attitudes [28]. Examples of these negative attitudes include the belief that Chinese Americans are "taking away too many jobs from Americans" and "passing on information to the Chinese government." One-fourth of the participants endorsed at least five out of a list of 12 negative stereotypes against Chinese Americans. More recently, Asian Americans are the target of verbal and physical assaults because they are often perceived as being responsible for the COVID-19 pandemic. Between March 2020 and August 2020, there were over 2500 reported incidents of coronavirus-related race-based assault against people of Asian descent in the USA [29]. Although this study focused only on attitudes toward one Asian ethnic group, it is likely that these negative attitudes also exist toward 
Asian Americans of other ethnicities, because mainstream America regards Asian Americans as homogenous and believes that differences among Asian ethnicities are nonexistent or unimportant [30]. When it is the providers who regard Asian Americans as a monolith, risk factors unique to particular Asian ethnic groups may be ignored.

Drawing from research on medical decision making for Black American patients, we hypothesize that providers' perceptions of Asian Americans patients have implications for patient-provider interactions and communication and for treatment decisions. We provide some illustrative examples below.

Model Minority Myth Asian Americans are now being positively stereotyped by mainstream America; however, this was not always the case in the history of the USA. From the $1800 \mathrm{~s}$ through the mid-1900s, Asians in the USA were regarded pejoratively as sinister and uncivilized interlopers of White American life [31]. Some of the terms [32] directed against those of Asian descent include "yellow perils," "menace," and "deviants". This narrative changed dramatically in the $1960 \mathrm{~s}$ with the rise of the struggle for civil rights among Black Americans and the attention that Black Americans drew to the racial injustice toward them. Within this context, the notion of Asian Americans as the "model minority" was born. In this new discourse [33], Asian Americans are "hard working, well educated, socioeconomically successful, studious, obedient, compliant with authority, and uncomplaining." The model minority myth implies that Asian Americans are more successful than other racial minority groups, that the success of Asian Americans is due to their own hard work, and that other racial minorities' lack of success was due to their laziness or incompetence [34]. Thus, White America pitted Asian Americans as a wedge against Black and other minority groups in order to justify continued racism in the USA [35].

Although race-based stereotypes with a negative valence are harmful, positively valenced stereotypes such as the model minority myth can also have deleterious effects on the targets. In a study of 291 Asian Americans [36], those who endorsed more positive stereotypes about Asian Americans-such as those that characterize the model minority myth - were more likely to report more physical and psychological distress, perhaps because these individuals believed they fell short of the model minority ideals.

Healthcare providers' explicit and implicit health-related stereotypes about Asian American patients may influence the providers' decisions about treatment, screening, and other procedures they recommend to these patients. Providers who subscribe to the model minority stereotype may underestimate Asian American patients' healthcare needs [37]. For example, Asian Americans are commonly perceived as being intelligent [30]; in particular, Asians are believed to be more intelligent than members of other racial minority groups. Providers may therefore assume that Asian American patients have a better understanding of technical explanations and treatment regimens than these patients actually do. Similarly, providers may assume that traditional Asian diets are healthful and may therefore omit dietary counseling with Asian American patients [38]. Healthcare providers may miss or ignore signs that some Asian patients are actually at increased mental health risk stemming from the model minority myth.

Racial Microaggressions Racial microaggressions are subtle, automatic, and unintentional insults and put-downs against a person because of their race [39]. There are several common forms of racial microaggressions that Asian Americans experience [30] which have implications for their healthcare. First, non-Asians often overlook interethnic differences among Asian subgroups. This perception is summarized in the common assertion, "All Asians look alike." Along a similar vein, although immigration status and histories of Asian Americans are varied, and a large proportion of Asian American families have lived in the US for generations, the general US population tends to perceive Asian Americans as "perpetual foreigners" or "aliens in their own land" [30], whereby Asians are automatically assumed to be foreign-born and not being truly "American." Indeed, Asian Americans report being told that they speak English well (the assumption being that English could not possibly be their first language) and being asked where they are from (with the questioner often responding incredulously when an Asian American says they are from a particular US city or state). When providers hold these misconceptions about Asian patients, they may neglect cultural, historical, and social differences among Asian ethnic groups, even though these differences play a role in determining health status and outcomes.

Second, providers may pathologize culturally based illness representations, such as the influence of the spirit in epilepsy [40] and the Chinese concept of heat (yang) and cold (yin) [41]. Asian communication styles may also be misconstrued, such that an Asian American patient who socially presents as being reticent may be viewed as being disinterested or unmotivated for further tests and treatment. Overall, microaggressions may jeopardize the patient-provider relationship, leading to patients feeling disrespected and distrustful.

Gender The term "racialized sexism," when applied to Asian American women, encapsulates the "intersectional discrimination that sets Asian American women apart from Asian American men as well as White women and other women of color" [42]. Asian American women have been exoticized and sexualized in mainstream American culture as mainly fulfilling the role of serving White men sexually. This trope stems from the enslavement of Asian women to be sex slaves, or comfort women, during the US wars in Asia, as well as from popular media's depiction of the Suzy Wong and the Dragon 
Lady [43, 44]. Asian American women continue to experience these stereotypes today. In a recent study, Asian American women participants shared that they are expected to be submissive sexual objects [30]. As one woman put it, "White men believe that Asian women are great girlfriends, wait hand and foot on men, and don't back-talk or give them shit." Racialized sexism of Asian American women has deleterious impact on their physical well-being [45]. It is not yet known what implications racialized sexism of Asian American women has on provider perceptions. Drawing from the literature on the healthcare experiences of Black American women [46], we speculate that the history of Asian American women in USA, as well as the ongoing stereotypes about these women's sexuality, will play a role in how providers deliver sexual and reproductive healthcare. For example, if providers believe that Asian American women are sexually conservative and wait until marriage to engage in sexual activity, they may neglect to offer counseling about contraceptives and safe sex practices.

Income and Poverty In addition to racial stereotypes, stereotypes based on socioeconomic status can play a role in provider perceptions. Americans tend to attribute poverty to shortcomings within the individual, such as laziness and lack of motivation, rather than to structural inequalities [47]. This worldview posits that poor people have no one to blame for their poverty but themselves. Healthcare providers who harbor similar attitudes toward the poor may interpret patient behaviors and predict treatment adherence negatively. This has implications for Asian Americans because, as described, significant pockets of the Asian American population live in poverty. A greater proportion of Asians (11.1\%) live at the poverty line compared with non-Hispanic White Americans (9.6\%) [48], with Hmong, Bangladeshi, and Cambodian being the poorest Asian ethnic groups [49]. With regard to access to healthcare, $6.6 \%$ of Asian Americans did not have health insurance in 2017, compared with $5.9 \%$ of non-Hispanic White Americans [48]. Furthermore, being poor contradicts the model minority myth that Asian Americans are of higher socioeconomic status. It is not yet known how Asian Americans who do not fit the model minority myth are perceived. It is possible that they are deemed inferior and defective ("Your people are so successful, so what's wrong with you?"). Research is needed to explore this possibility, as well as examine the implications for provider perceptions.

\section{Illusory Correlation}

People selectively attend to and interpret information that supports their preconceived stereotypes about those of specific social groups, thereby creating an illusory sense of accuracy about one's stereotypes when such accuracy does not exist [19]. In terms of medical decision making, a provider predicts the likelihood a patient would adhere to treatment based on that provider's preconceived beliefs about the social group to which the patient belongs (e.g., believing that older patients are more stubborn) and selectively attend to instances when those beliefs are supported and overlook instances when the beliefs are not supported [24].

Illusory correlations may take root during medical training if medical students and residents are placed in training hospitals set in low-income neighborhoods with disproportionately high numbers of racial minorities [18]. In those settings, trainees begin to associate health-related behaviors with certain demographic groups, even though these behaviors are due more to external circumstances, such as income, than to the patients' racial backgrounds. For example, racial minority patients may be more likely to decline recommended treatments due to apprehensions about costs or due to mistrust of medical professionals. Repeated interactions with patients perceived as being non-adherent or "difficult" may lead to providers' future reluctance to suggest intensive treatment for minority patients. When providers behave on the basis of their stereotypic expectations of patients, this can give rise to behavioral confirmation [50] or self-fulfilling prophecies, whereby providers behave in ways that elicit responses from the patient that affirm those stereotypes [19, 24]. Another contributor to sustaining illusory correlation is the ultimate attribution error [51], whereby a White provider attributes a White patient's behavior, such as treatment non-adherence, to situational factors (e.g., inability to pay) but attributes the same behavior in racial minority patient to dispositional factors (e.g., stubbornness) [24].

Illusory correlation has implications for Asian American patients, many of whom are of very low income and lack health insurance, and who may therefore avoid treatments and follow-ups that they cannot afford. Furthermore, due to the legacy of trauma and past USwar involvement in their countries of origin, as well as current experiences of racism in the USA, Southeast Asian Americans may mistrust their medical providers and be therefore wary of adhering to treatment recommendations [42].

\section{Cognitive Load}

Cognitive load [24] refers to "the amount of mental activity imposed on working memory, which may come from competing mental tasks, environmental factors, our own psychological or physiological state (e.g., fatigue), as well as from the demands inherent in the task at hand (i.e., intrinsic cognitive load)." Cognitive load negatively affects ability to consciously counter one's stereotypes and increases susceptibility to making decisions based on implicit bias [52]. As described below, providers are more susceptible to illusory correlations when medical decisions have to be made quickly, under time pressure, and with incomplete information [24]. 
People employ two cognitive pathways when making sense of social experience and deciding how to respond [53]. Dual process models [52] hypothesize two separate and interactive cognitive systems to make sense of social information. One cognitive system operates quickly and effortlessly and relies on unconscious biases. Although this system allows one to navigate their surroundings effortlessly and without having to pause and slowly process incoming information, it makes one susceptible to acting based on implicit biases and stereotypes based on information gathered quickly from interactions with others. The other system is activated more slowly and deliberately and is a conscious effort to make sense of the world in a more effortful and accurate way. This latter system is difficult to employ successfully when one is distracted and multitasking.

This dual-system conceptualization is important because although providers are assumed or expected to regard patients fairly and without bias, providers are in fact susceptible to relying on automatic and unconscious biases when interacting with minority patients [54]. This is the case even among providers who genuinely denounce racial stereotypes, because the automatic cognitive processing system is built upon years of socialization and social messages about race $[54,55]$. People can consciously and actively counter these stereotypes and biases if they choose to [56]. However, cognitive overload - caused by distraction, time pressure, multitasking, and anxiety - makes it more difficult to process social information deeply and increases susceptibility to reactions that are influenced by automatic stereotypes [54]. Furthermore, complex decisions, such as medical decision making involving diagnoses and treatment plan, are more likely to evoke stereotypes [19].

Asian Americans who are of low income or are uninsured are likely to seek medical care in healthcare facilities that serve low-income patients, which are the settings where providers experience cognitive overload. Burgess [24] proposed a list of ways that cognitive overload can lead to poor medical decisions. First, cognitive load impedes deliberate information processing and therefore leads to poor medical decisions and patient care. Second, the attenuated levels of controlled and deliberate information processing in turn increase the likelihood that providers would make medical decisions based on their racial stereotypes toward minority patients, thereby leading to poorer care and outcomes for these patients. Third, providers' cognitive loads are greater in healthcare settings where (i) there are greater number of competing demands; (ii) there are higher levels of time pressure, stress, and fatigue; (iii) there is a higher proportion of trainees; (iv) there is a lack of structural support for medical decision making; and (iv) there is a large proportion of racial minority patients. Finally, because racial minorities are more likely to seek care in settings where providers experience greater cognitive load, these patients are more likely to receive poorer healthcare than
White patients due to lower deliberate information processing and higher racial stereotypes by providers.

\section{Conclusion and Recommendations}

While there has been some research on how medical decision making is influenced by provider perceptions about Black and Latinx Americans, little is known about Asian Americans. Nonetheless, the present paper synthesized the social and cognitive mechanisms through which provider perceptions can have a deleterious impact on healthcare provided to Asian Americans. In order to enhance providers' cultural competence toward Asian American patients, multiple levels of interventions, from individual to structural, need to be taken. Non-Asians, including medical practitioners, tend to view all Asians as homogenous. This paper highlighted the differences across Asian ethnic groups and call on scholars and the medical field to be aware of these differences. Multicultural training about Asian Americans should note the diversity within this racial group, address stereotypes such as the model minority myth, and discuss microaggressions and indicate how they impair the patient-provider relationship. Such multicultural training needs to be based on evidence from what is known about whether and how these trainings are successful, including the effectiveness of interventions to reduce implicit bias $[57,58]$. Because Asian Americans, as well as other racial minority groups, are complex and diverse, the notion of "culture" needs to be expanded beyond race and includes other demographic and marginalized identities, including socioeconomic status. Broad structural changes [54] that make room for more patient-provider interaction are needed to minimize providers' cognitive load, especially in healthcare settings that serve underprivileged Asian Americans.

We offer the following research recommendations to further explore the role of provider perceptions in medical decision making for Asian American patients:

1) Broadly, there is a dearth of research on Asian Americans' experiences in healthcare settings. Drawing from the research protocols and findings of healthcare experiences of other groups of colors, researchers need to begin focusing on Asian Americans, especially providers' stereotypes and the potential impact of these stereotypes.

2) Although it is crucial to understand provider perceptions and biases, the provider-patient interaction does not occur in a vacuum. Rather, researchers need to acknowledge that, and study how, biases are systemic and inherent within clinics and hospitals, as well as within medical training curricula.

3) The literature highlights the importance of recognizing intersectionality of multiple marginalized identities. In 
additional to race, many Asian Americans occupy other marginalized identities associated with their gender, income, sexual orientation, and ability status. Research on provider perceptions need to take these intersecting identities into consideration.

Finally, high cognitive load negatively affects medical decision making for all marginalized groups, not just Asian Americans. Researchers need to continue building upon what is already known about how working under multiple distractions and time constraints affect providers' perceptions and medical decision making.

In conclusion, research and healthcare communities ought to begin to focus their efforts toward documenting and understanding the content and the degree of bias experienced by Asian American patients. These are necessary first steps toward reducing health disparities among Asian Americans.

\section{Compliance with Ethical Standards}

Conflict of Interest The authors declare that they have no conflict of interest.

Ethical Approval This article does not contain any studies with human participants or animals performed by any of the authors.

\section{References}

1. Lee E. The making of Asian America. New York: Simon \& Schuster; 2015.

2. Lopez G, Ruiz NG, Patten, E. Key facts about Asian Americans, a diverse and growing population. Available from http://www. pewresearch.org/fact-tank/2017/09/08/key-facts-about-asianamericans. Published 2017. Accessed November 20, 19.

3. Ramakrishnan K, Shah S. One out of 7 Asian immigrants is undocumented. Retrieved from http://aapidata.com/blog/asian-undoc1in7. Published 2017. Accessed January 15, 2020.

4. McCracken M, Olsen M, Chen MS, et al. Cancer incidence, mortality, and associated risk factors among Asian Americans of Chinese, Filipino, Vietnamese, Korean and Japanese ethnicities. CA Cancer J Clin. 2007;57:190-205.

5. Kocchar R, Cilluffo A. Income inequality in the U.S. is rising rapidly among Asians. Available from http://www.pewsocialtrends. org/2018/07/12/income-inequality-in-the-u-s-is-rising-mostrapidly-among-asians. Published 2018. Accessed August 22, 2019.

6. Center for Disease Control and Prevention. Asian Americans and Pacific Islanders and chronic Hepatitis B. Available from https:// www.cdc.gov/hepatitis/populations/api.htm. Published 2019. Accessed December 6, 2019.

7. Center for Disease Control and Prevention. Tuberculosis (TB): Asian population. Available from https://www.cdc.gov/tb/topic/ populations/tbinasians/default.htm. Published 2018. Accessed November 20, 2019.

8. U.S. Department of Health and Human Services (2016). Cancer and Asians/Pacific Islanders. Available from https://minorityhealth.hhs. gov/omh/browse.. sppx?lvl=4\&lvlid=46. Published 2016. Accessed January 18, 2020.
9. Ho IK, Dinh KT. Cervical cancer screening among Southeast Asian American women. J Immigr Minor Health. 2011;13:49-60.

10. Jose PO, Frank ATH, Kapphahn KI, Goldstein BA, Eggleston K, Hastings KG, et al. Cardiovascular disease mortality in Asian Americans. J Am Coll Cardiol. 2014;64:2486-94.

11. Agency for Healthcare Research and Quality. National Healthcare Quality and Disparities Reports. Available from https://www.ahrq. gov/sites/default/files/wysiwyg/research/findings/nhqrdr/2018qdr. pdf. Published 2018. Accessed August 24, 2020.

12. Sun, L. H. Why Asian Americans have diabetes but don't know it. Available from https://www.washingtonpost.com/news/to-yourhealth/wp/2015/09/10/why-asian-americans-have-diabetes-butdont-know-it/. Published 2015. Accessed October 31, 2019.

13. Chen MS. Cancer health disparities among Asian Americans: what we do and what we need to do. Cancer. 2005;104:2895-902.

14. Geiger HJ. Racial stereotyping and medicine: the need for cultural competence. Can Med Assoc J. 2001;164:1699-700.

15. van Ryn M, Fu SS. Paved with good intentions: do public health and human service providers contribute to racial/ethnic disparities in health? Am J Public Health. 2003;93:248-55.

16. National Academy of Medicine. Unequal treatment: what healthcare providers need to know about racial and ethnic disparities in healthcare. Available from https://www.nap.edu/resource/ 10260/disparities providers.pdf Published 2002. Accessed August 24, 2020.

17. Rathore SS, Lenert LA, Weinfurt KP, Tinoco A, Taleghani CK, Harless W, et al. The effects of patient sex and race on medical students' ratings of quality of life. Am J Med. 2000;108:561-6.

18. Betancourt JR, Ananeh-Firempong O. Not me! Doctors, decisions, and disparities in health care. Cardiovasc Rev Rep. 2004;25:105-9.

19. Bodenhausen GV. The role of stereotypes in decision-making processes. Med Decis Mak. 2005;25:112-8.

20. van Ryn M. Research on the provider contribution to race/ethnicity disparities in medical care. Med Care. 2002;1:I-140-51.

21. Ngo-Metzger Q, Legedza ATR, Phillips RS. Asian Americans' reports of their health care experiences: results of a national survey. J Gen Intern Med. 2004;19:111-9.

22. U.S. Department of Health and Human Services. Cultural competence in medical education: a guidebook for schools. Available from https://www.hrsa.gov/sites/default/files/culturalcompetence/ cultcomp.pdf . Published 2004. Accessed December 7, 2019.

23. Association of American Medical Colleges. Cultural competence education. Retrieved from https://www.aamc.org/download/54338/ data/. Published 2005. Accessed September 24, 2019.

24. Burgess DJ. Are providers more likely to contribute to healthcare disparities under high levels of cognitive load? How features of the healthcare setting may lead to biases in medical decision making. Med Decis Mak. 2010;30:246-57.

25. Green AR, Carney DR, Pallin DJ, et al. Implicit bias among physicians and its prediction of thrombolysis decisions for Black and White patients. Arch Intern Med. 2007;22:1231-8.

26. Hirsh AT, Hollingshead NA, Ashburn-Nardo L, Kroenke K. The interaction of patient race, provider bias, and clinical ambiguity on pain management decisions. J Pain. 2015;16:558-68.

27. Maddux WW, Galinsky AD, Cuddy AJC, Polifroni M. When being a model minority is good... and bad: realistic threat explains negativity toward Asian Americans. Pers Soc Psychol B. 2008;34:74 89.

28. Edsall TB. $25 \%$ of U.S. view Chinese Americans negatively, poll says. Available from https://www.washingtonpost.com/archive/ politics/2001/04/26/25-of-us-view-chinese-americans-negativelypoll-says/bf04c71b-2d50-443c-bdfc-04d9f969d7e9. Published 2001. Accessed October 13, 2019.

29. Asian Pacific Policy and Planning Council. Attacks against AAPI Community Continue to Rise During Pandemic. Available from

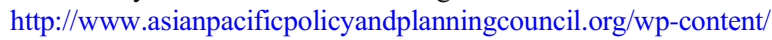


uploads/PRESS_RELEASE_National-Report_August27_2020. pdf. Published 2020. Accessed September 5, 2020.

30. Sue DW, Bucceri J, Lin AI, et al. Racial microaggressions and the Asian American experience. Asian Am J Psychol. 2009;S:88-101.

31. Wing JY. Beyond Black and White: the model minority myth and the invisibility of Asian American students. Urban Rev. 2007;39: 455-87.

32. Parks SJ, Yoo HC. Does endorsement of the model minority myth relate to anti-Asian sentiments among White college students? The role of a color-blind racial attitude. Asian Am J Psychol. 2016;7: 287-94.

33. Liu-Countryman J. The psychology of hate crimes as domestic terrorism: U.S. and global issues: volume two: assessment issues with victims and offenders. Santa Barbara, CA: Praeger; 2017. Chapter 3, The hard lessons of a model minority living in a racist world; p. 103-118.

34. Yoo HC, Burrola KS, Steger MF. A preliminary report on a new measure: internalization of the Model Minority Myth Measure (IM4) and its psychological correlates among Asian American college students. J Couns Psychol. 2010;57:114-27.

35. Kim CJ. The racial triangulation of Asian Americans. Polit Soc. 1999;27:105-38.

36. Gupta A, Szymanski DM, Leong FTL. The "model minority myth": internalized racialism of positive stereotypes as correlated of psychological distress, and attitudes toward help-seeking. Asian Am J Psychol. 2011;2:101-14.

37. Ibaraki AY, Hall GCN, Sabin JA. Asian American cancer disparities: the potential effects of model minority health stereotypes. Asian Am J Psychol. 2014;5:75-81.

38. Ho IK, Smith SA. Primary care providers' perceptions of young Cambodian American female patients. J Immigr Minor Health. 2018;20:1206-14.

39. Sue DW. Microaggressions in everyday life: race, gender, and sexual orientation. Wiley, Inc: Hoboken; 2010. 352p.

40. Fadiman A. The spirit catches you and you fall down: a Hmong child, her American doctors, and the collision of two cultures. New York: Farrar, Straus, and Giroux; 1997. 368p.

41. Wu Q, Liang X. Food therapy and medical diet therapy in traditional Chinese medicine. Clin Nutr Exp. 2018;18:1-5.

42. Mukkamala S, Suyemoto KL. Racialized sexism/sexualized racism: a multimethod study of intersectional experiences for Asian American women. Asian Am J Psychol. 2018;9:32-46.

43. Chan CS. Asian-American women: psychological responses to sexual exploitation and cultural stereotypes. Women Ther. 1988;6:338 .

44. Uchida A. The orientalization of Asian women in America. Women's Stud Int Forum. 1998;21:161-74.
45. Ho IK, Dinh KT, Bellefontaine SA, Irving AL. Sexual harassment and posttraumatic stress symptoms among Asian and White women. J Aggress Maltreat T. 2012;21:95-113.

46. Prather C, Fuller TR, Jeffries WL, et al. Racism, African American women, and their sexual and reproductive health: a review of historical and contemporary evidence and implications for health equity. Health Equity. 2018;2:249-59.

47. Wear D, Kuczewski MG. Medical students' perceptions of the poor: what impact can medical education have? Acad Med. 2008;83:639-45.

48. U.S. Department of Health and Human Services. Profile: Asian Americans. Available from https:/minorityhealth.hhs.gov/omh/ browse. aspx? $? \mathrm{lvl}=3 \&$ lvlid=63. Published 2019. Accessed December 28, 2019.

49. AAPI Data. Poverty. Available from https://aapidata.com/policy/ poverty/. Accessed January 15, 2020.

50. Claire T, Fiske ST. Intergroup cognition and intergroup behavior. In: Chapter 9, A systemic view of behavioral confirmation: counterpoint to the individualistic view. Mahwah: Lawrence Erlbaum Associates Publishers; 1998. p. 205-31.

51. Pettigrew TF. The ultimate attribution error: extending Allport's cognitive analysis of prejudice. Pers Soc Psychol B. 1979;5:46176.

52. Hogarth RM. Intuition: a challenge for psychological research on decision making. Psychol Inq. 2010;21:338-53.

53. Deutsch R, Strack F. Duality models in social psychology: from dual processes to interacting systems. Psychol Inq. 2006;17:16672.

54. Burgess DJ, Fu SS, van Ryn M. Why do providers contribute to disparities and what can be done about it? J Gen Intern Med. 2004;19:1154-9.

55. DiAngelo R. White fragility: why it's so hard for White people to talk about racism. Boston: Beacon Press; 2018. p. 2018, 192p.

56. Wegener DT, Dunn M, Tokusato D. Cognitive social psychology: the Princeton symposium on the legacy and future of social cognition. In: Chapter 17, The flexible correction model: Phenomenology and the use of naive theories in avoiding or removing bias. Mahwah: Lawrence Erlbaum Associates Publishers; 2001. p. 277-90.

57. Kenyon T, Beaulac G. Critical thinking education and debiasing. Informal Log. 2014;34:341-63.

58. Lai CK, Skinner AL, Cooley E, Murrar S, Brauer M, Devos T, et al. Reducing implicit racial preferences: II. Interventions effectiveness over time. J Exp Psychol Gen. 2016;145:1001-16.

Publisher's Note Springer Nature remains neutral with regard to jurisdictional claims in published maps and institutional affiliations. 